\title{
Macroinvertebrados bentónicos como indicadores de la calidad de agua en la microcuenca San Alberto, Oxapampa, Perú
}

\author{
Benthic macro invertebrates as water quality indicators in the San \\ Alberto micro basin, Oxapampa, Perú
}

Stefany Salcedo Gustavson', Lourdes Artica Cosme², Florencia Andrea Trama ${ }^{3}$ Universidad Continental

\section{RESUMEN}

Objetivos: Evaluar la biodiversidad de macroinvertebrados y la calidad ecológica de la microcuenca San Alberto (provincia de Oxapampa, Pasco) de abril a julio de 2013. Métodos: El muestreo fue realizado en tres zonas de la microcuenca San Alberto (cuenca alta, media y baja). En los tres sitios fueron colectados macroinvertebrados bentónicos en piedra y arena. Luego se estimaron: el índice biótico andino (IBA), de hábitat fluvial (IHF), de calidad de vegetación de ribera andina (QBRAnd) y de estado ecológico (ECOSTRIAND). Se evaluaron parámetros fisicoquímicos, nutrientes y metales en el agua. Resultados: Se registraron un total de 123 taxones de 47 familias (101 taxones en la cuenca alta, 77 en la cuenca media y 55 en la cuenca baja). La mayor abundancia de macroinvertebrados se presentó en la cuenca media. El IBA resultó ser de "muy buena" calidad para la cuenca alta, media y baja, pero al combinarse este índice con el QBR-And, para elaborar el Índice ECOSTRIAND, se observó el estado ecológico de las cuencas alta y media fueron de "muy buena" calidad y de la cuenca baja fue "regular". El método de muestreo de limpieza de piedras fue el que permitió colectar la mayor cantidad de taxones. Las mejores condiciones de hábitat fluvial, de vegetación ribereña y de estado ecológico, tras la aplicación de los índices IHF, QBR-And y ECOSTRIAND, se encuentran en la cuenca alta. Conclusiones: Los resultados indican que la diferencia de calidad de hábitat ribereño y fluvial así como de conductividad, sólidos disueltos y nitratos influyen negativamente sobre la

1 Bachiller en Ingeniería Ambiental de la Universidad Nacional Daniel Alcides Carrión, Pasco. Becaria de la Universidad Continental. 2 M.Sc., docente de la Universidad Nacional Daniel Alcides Carrión.

3 M.Sc., directora del Centro Neotropical de Entrenamiento en Humedales-Perú.

calidad del agua, y ésta, sobre la comunidad de macroinvertebrados. Las familias reportadas como sensibles a la contaminación (Leptophlebiidae, Oligoneuriidae, Perlidae, Anomalopsychidae, Calamoceratidae, Helicopsychidae, Odontoceridae, Blephariceridae) mostraron ser bioindicadores de calidaddeaguaparalamicrocuenca.

Palabras clave: Calidad de agua, bioindicador, macroinvertebrados bentónicos, monitoreo biológico, microcuenca, río San Alberto. 


\section{ABSTRACT}

Objetive: To assess the macro invertebrates' biodiversity and the ecological quality in the San Alberto micro basin (Oxapampa province, Pasco) from April to July 2013. Methods: Sampling was realized in three areas of the San Alberto basin (upper, middle and lower micro basin). In the three sites benthic macroinvertebrates in stone and sand were collected. The Andean biotic index $(A B I)$, fluvial habitat index (IHF), Riparian-Andean vegetation quality index (QBR-And) and ecological status index (ECOSTRIAND) were estimated. Also physicochemical parameters, nutrients and metals in water were estimated. Results: We recorded a total of 123 taxa from 47 families (101 taxa in the upper basin, 77 in the middle basin and 55 in the lower basin). The higher abundance of macro invertebrates was presented in the middle basin. The ABI presented "Very good water quality" for the three areas in the basin. However, when combining the $A B I$ with the QBR-And to elaborate the ECOSTRIAND index, the ecological status of the high and media basins were "very good quality" and the lower basin was "regular". The stones cleaning's sampling method allowed to collect the higher quantity of taxa. The best conditions of fluvial habitat, riparian vegetation and ecological status, after the application of the IHF, QBR-And and ECOSTRIAND indexes' were found in the upper basin. Conclusions: The results indicate that the quality difference between riverside and fluvial habitat and the conductivity, dissolved solids and nitrates affect negatively on the water quality, and this, on the macro invertebrates' community. The reported families as pollution sensitive (Leptophlebiidae, Oligoneuriidae, Perlidae, Anomalopsychidae, Calamoceratidae, Helicopsychidae, Odontoceridae, Blephariceridae) showed to be water quality's biomarkers for the micro basin.

Keywords: Water quality, biomarker, macro invertebrates, benthic, biological monitoring, San Alberto River.

\section{INTRODUCCIÓN}

Los sistemas fluviales son sometidos a presiones de uso afectando la calidad del agua, principalmente por ingreso de material particulado, actividades agrícolas, deforestación, fragmentación del hábitat, cambio del sustrato por remoción y extracción de materiales (1). El río San Alberto es la principal fuente de agua para consumo humano del distrito de Oxapampa y tiene sus nacientes dentro del Parque Nacional Yanachaga Chemillén (PNYCh) en la parte del bosque de neblina de la zona (2). Sin embargo, esta microcuenca, es perturbada por actividades humanas en la zona de amortiguamiento, las cuales podrían estar afectando la calidad de las aguas de las que depende la ciudad de Oxapampa, que es la capital del distrito y a la vez de la provincia del mismo nombre.

El estado ecológico es una medida de la calidad de las aguas, considerando a la cuenca hidrográfica como unidad de estudio (3). Integra información sobre el bosque de ribera y las áreas adyacentes, el canal y el lecho del río (características hidromorfológicas) y los organismos que los habitan (4). Desde los años 50, las evaluaciones biológicas están complementando a las medidas químicas $(5,6,7)$. La evaluación del recurso agua en la actualidad se ha complementado con monitoreos biológicos, en muchos casos con la utilización de macroinvertebrados bentónicos como indicadores de calidad de agua, lo cual permite detectar condiciones ambientales específicas y los cambios producidos por la contaminación (8).

En la microcuenca San Alberto, no se han realizado estudios de calidad de agua utilizando macroinvertebrados, por lo que el objetivo de la investigación fue identificar la estructura y composición de macroinvertebrados bentónicos de esta microcuenca y determinar la calidad ecológica con el índice biótico andino (IBA), resultados que serán utilizados como base para futuras investigaciones, acciones de manejo y para difundir la importancia de 
los sistemas de monitoreo biológico del agua a través de índices de calidad como el IBA, el IHF, el Qbr-And y el ECOSTRIAND.

Este estudio predice que la estructura y composición de macroinvertebrados bentónicos varía de acuerdo con la calidad ecológica y del agua de la microcuenca San Alberto, y que específicamente la calidad de agua será mejor en la cuenca alta que en la media y en la baja, por causa de la perturbación humana fuera del PNYCh.

\section{MATERIAL Y MÉTODOS}

\section{Área de estudio}

La microcuenca San Alberto se ubica en el distrito de Oxapampa (provincia del mismo nombre, departamento de Pasco), con sus nacientes dentro del PNYCh, una precipitación anual de 2502 mm,

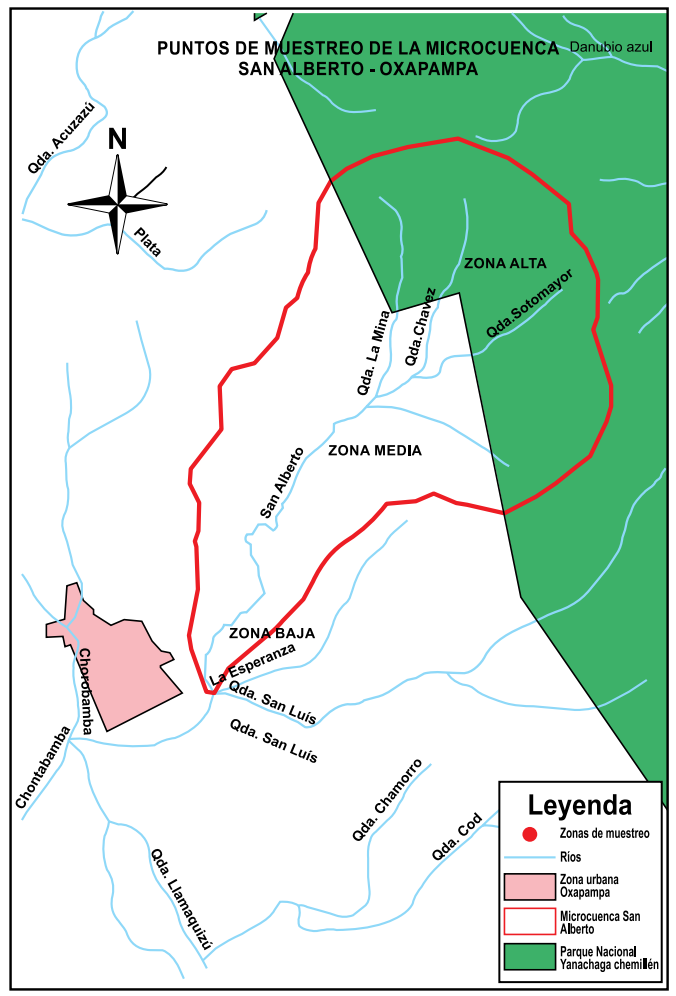

Figura $\mathrm{N}^{\circ}$ 1: Mapa de ubicación de los puntos de muestreo en la microcuenca San Alberto en Oxapampa, departamento de Pasco, Perú. una temperatura anual media de $13{ }^{\circ} \mathrm{C}$, y dos épocas, lluviosa (octubre-abril) y seca (mayo-septiembre) (9). Su pendiente es escarpada $\left(30-80^{\circ}\right)$ e inestable, con derrumbes frecuentes y erosión intensa. Se encuentra en la zona de vida Bosque Muy Húmedo Premontano Tropical (10).

\section{Zonas de muestreo}

El estudio definió tres zonas de muestreo (figura $\mathrm{N}^{\circ}$ 1). La cuenca alta (X; 460052 Y; 8835391), dentro del PNYCh, con bosques neblina de especies nativas (11). El punto situado en la cuenca media (X; 458064 Y; 8832829), dentro de un bosque secundario y cerca al camino principal. El punto situado en la cuenca baja (X; 457509 Y; 8831291), ubicado en el tramo urbano del sector San Alberto a 200 metros aguas arriba de la captación de agua para consumo humano.

\section{Muestreo}

Los muestreos fueron realizados de abril a julio de 2013 (estación seca). En cada zona de muestreo (cuenca) se evaluaron 20 metros lineales para determinar la calidad del hábitat fluvial a partir del índice IHF (12) y la calidad de la vegetación de ribera a partir del índice QBR-And (4). Valores por debajo de 40 para el IHF indican serias limitaciones para el desarrollo de una comunidad bentónica diversa, lo óptimo es superior a 75. En el caso del QBR-And se puede obtener una puntuación mínima de 25 y un máximo 100 para una ribera perfectamente conservada.

\section{Muestreo de macroinvertebrados}

El diseño fue completamente aleatorio, con tres tratamientos (cuencas), estratificación con dos sustratos (arena y piedras), y tres muestras de cada tipo de sustrato-método, en cuatro meses. El sustrato arena se evaluó con una red surber de $0.9 \mathrm{~m} 2$, colocando la red en sentido contrario al flujo del agua, con el cuadro de metal en la zona de arena. Luego, se removió el fondo dentro del cuadro de metal con la mano para remover 
el sustrato y los macroinvertebrados sean arrastrados por la corriente y queden atrapados en la red. Inmediatamente después se traspasó la muestra a un envase plástico (5).

El segundo método de colecta consistió en la limpieza de piedras $y$, al igual que en el método anterior, se realizaron tres repeticiones en cada zona de muestreo durante los cuatro meses de investigación. En este caso se delimitó $1 \mathrm{~m} 2$ del lecho del río y se procedió a extraer y lavar las piedras encontradas en el área. El lavado de piedras fue realizado dentro de un balde lleno de agua con ayuda de un cepillo, luego se procedió a tamizar el contenido del balde para almacenar el concentrado en un frasco de plástico. Las muestras colectadas (72 en total para los cuatro meses y las tres zonas de la cuenca) fueron fijadas en alcohol $70 \%$. Los organismos fueron separados e identificados a nivel de género por medio de un estereoscopio XTL-500 de 10-40X y de claves taxonómicas $(3,13,14,15)$. Se estimó la riqueza de familias, riqueza de taxones y abundancia (total y media) de individuos. El estudio utilizó el índice de abundance-based coverage estimator (ACE) para estimar el número de especies esperadas, el índice biótico andino (IBA) para evaluar la calidad de agua en cada zona de muestreo y el índice ECOSTRIAND, para determinar el estado ecológico de las zonas evaluadas en el río San Alberto, mediante la combinación de los resultados del IBA y el QBR-And.

\section{Muestreo de parámetros fisicoquímicos}

Los siguientes parámetros se tomaron por triplicado: temperatura, conductividad eléctrica y oxígeno disuelto por medio de un equipo multiparámetro marca YSI 85, el potencial de hidrógeno $(\mathrm{pH})$ y los sólidos totales disueltos (STD) usando el equipo $\mathrm{HI}$ 98129 marca HANNA, la turbidez utilizando el kit LaMotte Turbidity modelo TTM, y la profundidad con una regla de metal.

\section{Muestras de agua}

Se colectaron y almacenaron muestras de agua en frascos de vidrio color ámbar en cada zona de la cuenca y por cada fecha muestreo. Luego se transportaron al laboratorio para la determinación de nutrientes (nitrógeno total, fósforo total, sales solubles) y metales (cadmio, cobre, cromo, hierro, magnesio, zinc, plomo).

\section{RESULTADOS}

\section{Índices de calidad de hábitat y vegetación de ribera}

Los valores de los índices de hábitat fluvial (IHF) y de vegetación de quebrada andina (QBR-And) estimados en cada zona de muestreo y las puntuaciones de referencia obtenidos se muestran en la tabla $N^{\circ} 1$. Ambos índices mostraron sus mayores valores en la zona alta, luego en la zona media y por último en la zona baja.

El índice IHF en la cuenca alta tuvo un valor de 75, con alta frecuencia de rápidos; sustrato compuesto por bloques, piedras, cantos, gravas no fijadas por sedimentos finos, en menor grado se encontró arena, limo y arcilla; regímenes de velocidad con dos categorías, rápido-profundo y rápidosomero; cauce sombreado con ventanas. Presencia de hojarasca, raíces expuestas, troncos, ramas y diques naturales con la

Tabla $N^{\circ}$ 1: Valores de los índices de cada zona de muestreo de la microcuenca San Alberto.

\begin{tabular}{lcccc}
\hline \multicolumn{1}{c}{ Zona } & Altitud & IHF & QBR-And & Color de QBR-And \\
\hline Zona alta & 2376 & 75 & 100 & Azul \\
Zona media & 1963 & 52 & 85 & Verde \\
Zona baja & 1905 & 32 & 30 & Naranja \\
\hline
\end{tabular}


cobertura vegetal acuática compuesta por briofitos y en menor grado por el pecton.

La cuenca media obtuvo un valor de 52 con escasa frecuencia de rápidos; sustrato con bloques, piedras, cantos, gravas y poco fijadas por sedimentos finos, en menor grado se encontró arena, limo y arcilla; regímenes de velocidad con dos categorías, rápido-profundo y rápido-somero; cauce con grandes claros. Presencia de hojarasca, troncos y ramas con cobertura vegetal acuática representada por el pecton.

La cuenca baja obtuvo un valor de 32, con ocurrencia ocasional de rápidos; sustrato de cantos y gravas fijadas por sedimentos finos, con arena, limo y arcilla; régimen de velocidad de una sola categoría, rápidoprofundo; cauce totalmente expuesto. Elementos de heterogeneidad con poca hojarasca con cobertura vegetal acuática de pecton, pero en menor grado que en la cuenca media. Ninguna de las zonas de la cuenca presentó variaciones de condiciones de hábitat fluvial a lo largo de la investigación.

El índice QBR-And en la cuenca alta obtuvo una puntuación de 100, de "calidad muy buena", con más del $80 \%$ de la zona de ribera con cubierta vegetal y especies autóctonas sin alteraciones. La cuenca media obtuvo 85, considerada dentro del rango de "calidad buena", con ligera perturbación. La cuenca baja, en cambio, obtuvo una puntuación de 30 , la más baja y cuyo rango es considerado de "mala calidad", por tener una fuerte alteración de la ribera, poca cubierta vegetal y especies introducidas (Eucalyptus spp., Pinus spp.) (tabla $\mathrm{N}^{\circ} 1$ ).

\section{Macroinvertebrados bentónicos}

La riqueza total de macroinvertebrados colectados en las tres zonas de muestreo durante todo el periódo de investigación fue de 123 taxones en 47 familias y 13 órdenes. En la cuenca alta fueron registrados 101 taxones (de 42 familias y 10 órdenes); en Tabla $N^{\circ}$ 2: Estimado para cada cuenca y tipo de sustrato según el índice ACE.

\begin{tabular}{|c|c|c|c|}
\hline \multirow{2}{*}{ Cuenca } & \multirow{2}{*}{ Sustrato } & \multicolumn{2}{|c|}{ Taxones } \\
\hline & & Observados & Esperados \\
\hline \multirow{2}{*}{ Alta } & Piedra & 98 & 108 \\
\hline & Arena & 45 & 56 \\
\hline \multirow{2}{*}{ Media } & Piedra & 68 & 73 \\
\hline & Arena & 20 & 33 \\
\hline \multirow{2}{*}{ Baja } & Piedra & 52 & 57 \\
\hline & Arena & 9 & 36 \\
\hline
\end{tabular}

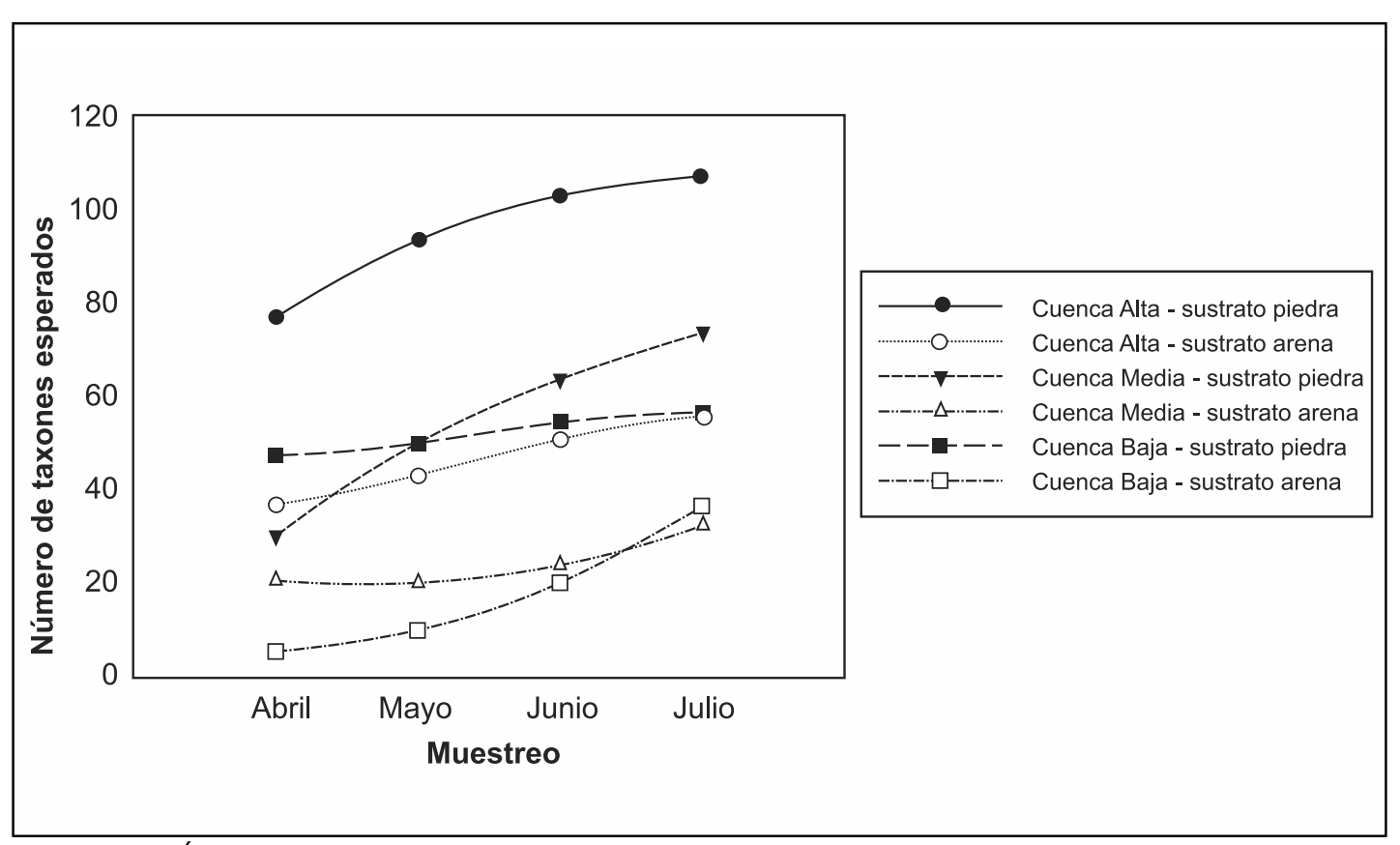

Figura $N^{\circ}$ 2: Índice ACE estimado para cada cuenca y tipo de sustrato. 
Tabla $N^{\circ}$ 3: Presencia de taxones de macroinvertebrados en cada parte de la cuenca evaluada y por tipo de sustrato evaluado.

\begin{tabular}{|c|c|c|c|c|c|c|c|c|}
\hline \multirow{2}{*}{$\begin{array}{l}\text { Clase/ } \\
\text { Orden }\end{array}$} & \multirow{2}{*}{ Familia } & \multirow{2}{*}{ Taxones } & \multicolumn{2}{|c|}{ Zona alta } & \multicolumn{2}{|c|}{ Zona media } & \multicolumn{2}{|c|}{ Zona baja } \\
\hline & & & Piedra & Arena & Piedra & Arena & Piedra & Arena \\
\hline \multirow[t]{2}{*}{ Hemiptera } & Veliidae & Rhagovelia sp. & $\mathrm{x}$ & $x$ & & & & \\
\hline & $\begin{array}{l}\text { Macroveliidae } \\
\text { Hydrometridae }\end{array}$ & $\begin{array}{l}\text { Chepuvelia sp. } \\
\text { Veliometra sp. }\end{array}$ & & $x$ & & & $\mathrm{x}$ & $x$ \\
\hline \multirow[t]{2}{*}{ Lepidoptera } & Pyralidae & Petrophila sp. & $\mathrm{x}$ & & & & & \\
\hline & $\begin{array}{l}\text { Noctuidae } \\
\text { Tortricidae }\end{array}$ & $\begin{array}{l}\text { Parapoynx sp. } \\
\text { Bellura sp. } \\
\text { Archips sp. }\end{array}$ & $\begin{array}{l}x \\
x\end{array}$ & & & & & $\mathrm{x}$ \\
\hline \multirow[t]{9}{*}{ Ephemeroptera } & Leptohyphidae & Leptohyphes sp. & $\mathrm{x}$ & $\mathrm{x}$ & $\mathrm{x}$ & $\mathrm{x}$ & $\mathrm{x}$ & \\
\hline & & Haplohyphes sp. & $\mathrm{x}$ & $\mathrm{x}$ & & & & \\
\hline & Baetidae & Baetodes sp. & $\mathrm{x}$ & $\begin{array}{l}x \\
x\end{array}$ & $\mathrm{x}$ & $\mathrm{x}$ & $\mathrm{x}$ & $\mathrm{x}$ \\
\hline & & Mayobaetis sp. & $x$ & & & & & \\
\hline & & Zelusia sp. & $\mathrm{x}$ & $\mathrm{x}$ & $\mathrm{x}$ & & $x$ & \\
\hline & & Nanomis sp. & $\mathrm{x}$ & $\mathrm{x}$ & $\mathrm{x}$ & $x$ & $x$ & \\
\hline & & Andesiops sp. & $\mathrm{x}$ & $x$ & $x$ & $\mathrm{x}$ & $x$ & $x$ \\
\hline & Leptophlebiidae & Thraulodes sp. & $\mathrm{x}$ & $\mathrm{x}$ & $x$ & & $\mathrm{x}$ & \\
\hline & Oligoneuriidae & Lachlania sp. & $\mathrm{x}$ & & & & & \\
\hline Odonata & Gomphidae & $\begin{array}{l}\text { sp. } 1 \\
\text { sp. } 2\end{array}$ & $\mathrm{x}$ & & & & $\mathrm{x}$ & \\
\hline \multirow[t]{16}{*}{ Coleoptera } & Elmidae & Macrelmis sp. & $x$ & $x$ & $\mathrm{x}$ & $\mathrm{x}$ & & \\
\hline & & Heterelmis sp. & $x$ & & $x$ & $\mathrm{x}$ & $x$ & \\
\hline & & Austrolimnius sp. & $x$ & $x$ & $x$ & & & \\
\hline & & Onychelmis sp. & $x$ & & & & & \\
\hline & & Neoelmis sp. & $\mathrm{x}$ & & & $\mathrm{x}$ & & \\
\hline & & Hexacylloepus sp. & $\mathrm{x}$ & $\mathrm{x}$ & $\mathrm{x}$ & & $x$ & \\
\hline & & Phanoceroides sp 1 & $x$ & & $x$ & & $\mathrm{x}$ & \\
\hline & & Phanoceroides sp. 2 & $\hat{x}$ & & $\mathrm{x}$ & & $x$ & \\
\hline & & Stenelmis sp. & & & $x$ & & $x$ & \\
\hline & & Disersus sp. & $x$ & $\mathrm{x}$ & $x$ & $x$ & & \\
\hline & & Pseudodisersus sp. & $x$ & & $x$ & & & \\
\hline & Psephenidae & Pheneps sp. & $x$ & & $x$ & $\mathrm{x}$ & & \\
\hline & Scirtidae & Elodes sp. & $\mathrm{x}$ & & & & & \\
\hline & & Scirtes sp. & $\mathrm{x}$ & & & $\mathrm{x}$ & & \\
\hline & Ptılodactylıdae & Anchytarsus sp. & $\mathrm{x}$ & & & & & \\
\hline & Hydrophiloidea & Phaenonotum sp. & $\begin{array}{c}x \\
y\end{array}$ & $\mathrm{x}$ & $\mathrm{x}$ & $\mathrm{x}$ & & \\
\hline \multirow{26}{*}{$\begin{array}{l}\text { Plecoptera } \\
\text { Megaloptera } \\
\text { Trichoptera }\end{array}$} & Perlidae & Anacroneuria sp. & $x$ & $\mathrm{x}$ & $\mathrm{x}$ & $\mathrm{x}$ & $x$ & \\
\hline & Corydalidae & Corydalus sp. & & & $x$ & $\mathrm{x}$ & $x$ & \\
\hline & Hydroptilidae & Neotrichia sp. & $\mathrm{x}$ & $x$ & & & & \\
\hline & & Ceratotrichiasp. & & & $x$ & & & \\
\hline & & Anchitrichia sp. & & & $\mathrm{x}$ & & & \\
\hline & & Ochrotrichia sp. & $\mathrm{x}$ & & $x$ & $x$ & $x$ & \\
\hline & & Alisotrichia sp. 1 & $x$ & & $\mathrm{x}$ & $\mathrm{x}$ & $x$ & \\
\hline & & Alisotrichia sp.2 & & & $x$ & & $x$ & \\
\hline & & Alisotrichia sp.3 & & & & & $x$ & \\
\hline & & sp. 1 & $\mathrm{x}$ & & & $\mathrm{x}$ & & \\
\hline & & sp.2 & $x$ & & & & & \\
\hline & & sp.3 & $x$ & $x$ & $x$ & & $x$ & \\
\hline & & Dibusa sp. & $\mathrm{x}$ & & & & & \\
\hline & Leptoceridae & Grumichella sp. 1 & $x$ & $x$ & & $x$ & $x$ & \\
\hline & & Grumichella sp.2 & $x$ & & $x$ & & & \\
\hline & & Nectopsyche sp. 1 & $x$ & $\mathrm{x}$ & & & & \\
\hline & & Brachicetodes sp. & $x$ & $x$ & $x$ & & & \\
\hline & & Triplectides sp. & $x$ & & & & & \\
\hline & & Atanatolica sp. & $x$ & $\mathrm{x}$ & $x$ & & & \\
\hline & & Oecetis sp. & $x$ & & & & & \\
\hline & Hydropsychidae & Smicridea sp. 1 & $x$ & & & & $x$ & \\
\hline & & Smicridea sp.2 & $x$ & & $x$ & $x$ & $x$ & \\
\hline & & Smicridea sp.3 & $x$ & $x$ & $x$ & & $x$ & \\
\hline & & Leptonema sp. 1 & & & $x$ & $x$ & $x$ & \\
\hline & & Leptonema sp. 2 & $x$ & & $x$ & $x$ & $x$ & \\
\hline & & Leptonema sp.3 & & & $x$ & & & \\
\hline
\end{tabular}


Continua Tabla $N^{\circ} 3$.

\begin{tabular}{|c|c|c|c|c|c|c|c|c|}
\hline \multirow{2}{*}{$\begin{array}{l}\text { Clase/ } \\
\text { Orden }\end{array}$} & \multirow{2}{*}{ Familia } & \multirow[b]{2}{*}{ Taxones } & \multicolumn{2}{|c|}{ Zona alta } & \multicolumn{2}{|c|}{ Zona media } & \multicolumn{2}{|c|}{ Zona baja } \\
\hline & & & Piedra & Arena & Piedra & Arena & Piedra & Arena \\
\hline \multirow[t]{14}{*}{$\cdots$} & \multirow[t]{3}{*}{ Glossosomatidae } & \multirow{3}{*}{$\begin{array}{l}\text { Mortoniella sp. } \\
\text { Culoptila sp. } 1 \\
\text { Culoptila sp.2 }\end{array}$} & $x$ & \multirow[t]{2}{*}{$x$} & $x$ & \multirow[t]{3}{*}{$x$} & \multicolumn{2}{|l|}{$x$} \\
\hline & & & $x$ & & \multirow[t]{2}{*}{$x$} & & \multirow[t]{3}{*}{$x$} & \\
\hline & & & $x$ & $x$ & & & & \\
\hline & Anomalopsychidae & Contulma sp. & $x$ & $x$ & & & & \\
\hline & Calamoceratidae & Phylloicus sp. & $x$ & $x$ & $x$ & $x$ & & \\
\hline & Helicopsychidae & Helicopsyche sp. & $x$ & & $x$ & $x$ & $x$ & \\
\hline & Philopotamidae & Chimarra sp. & $x$ & & & & $x$ & \\
\hline & Odontoceridae & Marilia sp. & $x$ & & $x$ & & & \\
\hline & & Anastomoneura sp. & $x$ & $x$ & & & & \\
\hline & Polycentropodidae & Polycentropus sp. & $x$ & & $x$ & & $x$ & \\
\hline & & Certonina sp. & & & $\mathrm{x}$ & $x$ & & \\
\hline & Xiphocentronidae & Xiphocentron sp. & $x$ & & $x$ & $x$ & $x$ & \\
\hline & Sericostomatidae & Grumicha sp. & $x$ & $x$ & $x$ & $x$ & $x$ & \\
\hline & Hydrobiosidae & Atopsyche sp. & $x$ & $x$ & $x$ & $x$ & $x$ & \\
\hline Diptera & Blephariceridae & Limonicola sp. 1 & $x$ & & $x$ & & & \\
\hline & & Limonicola sp. 2 & & & $x$ & & & \\
\hline & & Paltostoma sp. & & & $x$ & $x$ & & \\
\hline & Ceratopogonidae & Alluaudomyia sp. & $x$ & $x$ & & & & \\
\hline & & Atrichopogon sp. 1 & $x$ & $x$ & & & & \\
\hline & & Atrichopogon sp.2 & $x$ & & & & & \\
\hline & & Atrichopogon sp.3 & $x$ & & & & $x$ & \\
\hline & & Atrichopogon sp.4 & $x$ & & & & & \\
\hline & Chironomidae & $\begin{array}{l}\text { Forcipomyia sp. } \\
\text { Podonominae }\end{array}$ & $x$ & & & & & \\
\hline & & Podonomus sp. & $x$ & $x$ & $x$ & $x$ & $x$ & \\
\hline & & $\begin{array}{l}\text { Podonomopsis sp. } \\
\text { Tanypodinae }\end{array}$ & $x$ & $x$ & $x$ & & $x$ & \\
\hline & & sp. 1 & $x$ & $x$ & & $x$ & $x$ & $x$ \\
\hline & & sp.2 & $x$ & $x$ & $\mathrm{x}$ & $x$ & $x$ & $x$ \\
\hline & & sp.3 & $x$ & $x$ & $x$ & $x$ & $x$ & \\
\hline & & Chironominae & & & & & & \\
\hline & & $\begin{array}{c}\text { Rheotanytarsus sp. } \\
\text { Orthocladiinae }\end{array}$ & $x$ & $x$ & $x$ & $x$ & $x$ & \\
\hline & & Cricotopus sp. & $x$ & $x$ & $x$ & $x$ & $x$ & $x$ \\
\hline & Simulidae & Simulium sp. & $x$ & $x$ & $x$ & $x$ & $x$ & $x$ \\
\hline & Empididae & Chelifera sp. & $x$ & $x$ & $x$ & $\mathrm{x}$ & $x$ & \\
\hline & & Neoplasta sp. & $x$ & & $x$ & $x$ & & $x$ \\
\hline & Psychodidae & Maruina sp. & $x$ & & $x$ & $x$ & $x$ & \\
\hline & & Telmatoscopus sp. & $x$ & $x$ & & & $x$ & \\
\hline & & Psychoda sp. & $x$ & & & & & \\
\hline & Dixidae & Dixella sp. & $x$ & & $x$ & & & \\
\hline & Tipulidae & Limonia sp. & $x$ & & $x$ & & $x$ & \\
\hline & & Pseudolimnophila sp. & $x$ & & & & & \\
\hline & & Megistocera sp. & $x$ & $x$ & & $x$ & & \\
\hline & & Tipula sp. & $x$ & & $x$ & & & \\
\hline & & Molophilus sp. & & $x$ & & $x$ & & \\
\hline & & Hexatoma sp. & & $\mathrm{x}$ & & $x$ & & \\
\hline & Stratiomyidae & sp. 1 & $\mathrm{x}$ & & & & & \\
\hline & Muscidae & Limnophora sp. & & & & $\mathrm{x}$ & & \\
\hline & Ephidridae & sp. 1 & $\mathrm{x}$ & & & & & \\
\hline & Thaumalidae & sp.1 & $\mathrm{x}$ & & & & & \\
\hline Collembola & Entomobryidae & sp. 1 & & $\mathrm{x}$ & & & & \\
\hline & & sp.2 & $\mathrm{x}$ & & & & & \\
\hline & Hypogastrudidae & sp. 1 & & & & $\mathrm{x}$ & & \\
\hline Acari/Acarina & Acaridae & sp. 1 & $\mathrm{x}$ & & $\mathrm{x}$ & & $\mathrm{x}$ & \\
\hline & & sp. 2 & & & $x$ & & $\mathrm{x}$ & \\
\hline & & sp. 3 & & & $\mathrm{x}$ & & & \\
\hline & & sp. 4 & & & & & $\mathrm{x}$ & \\
\hline & & sp. 5 & & & & & $x$ & \\
\hline & & $\begin{array}{l}\text { sp. } 6 \\
\text { sn } 7\end{array}$ & & & & $\begin{array}{l}x \\
x\end{array}$ & $\mathrm{x}$ & \\
\hline Amphipoda & Hyalellidae & Hyallela sp. & & & $\mathrm{x}$ & & & \\
\hline Isopoda & & sp. 1 & & & $x$ & & & \\
\hline & & & 93 & 46 & 64 & 45 & 53 & 9 \\
\hline
\end{tabular}


la cuenca media, 77 (de 32 familias y 10 órdenes); y en la cuenca baja, 55 (de 27 familias y 10 órdenes) evidenciando que algunos taxones se compartieron entre las diferentes zonas. El método de colecta por sustrato de piedra tuvo más taxones colectados ( 113 taxones de 44 familias y 13 órdenes), en comparación con el aplicado en arena (52 taxones de 27 familias y 9 órdenes). El índice ACE estimado aumentó en todos los muestreos (tabla $\mathrm{N}^{\circ} 2$, figura $N^{\circ} 2$ ). Sin embargo, el sustrato de piedra siempre presentó mayor número de taxones esperados que el sustrato de arena y fue mayor en la cuenca alta en comparación con la media y baja (tabla $\mathrm{N}^{\circ} 3$ ).

\section{Riqueza media de familias y taxones}

La figura $\mathrm{N}^{\circ} 3$ muestra la riqueza de las familias y taxones identificados en la investigación. La riqueza media de familias de macroinvertebrados fue mayor en la cuenca alta que en la media y la baja ( $F=70.54 ; \quad g l=2,71 ; \quad P=0,0001$ ) (figura $\mathrm{N}^{\circ} 3 \mathrm{a}$ ). Asimismo, la misma variable fue mayor al utilizar el sustrato de piedra

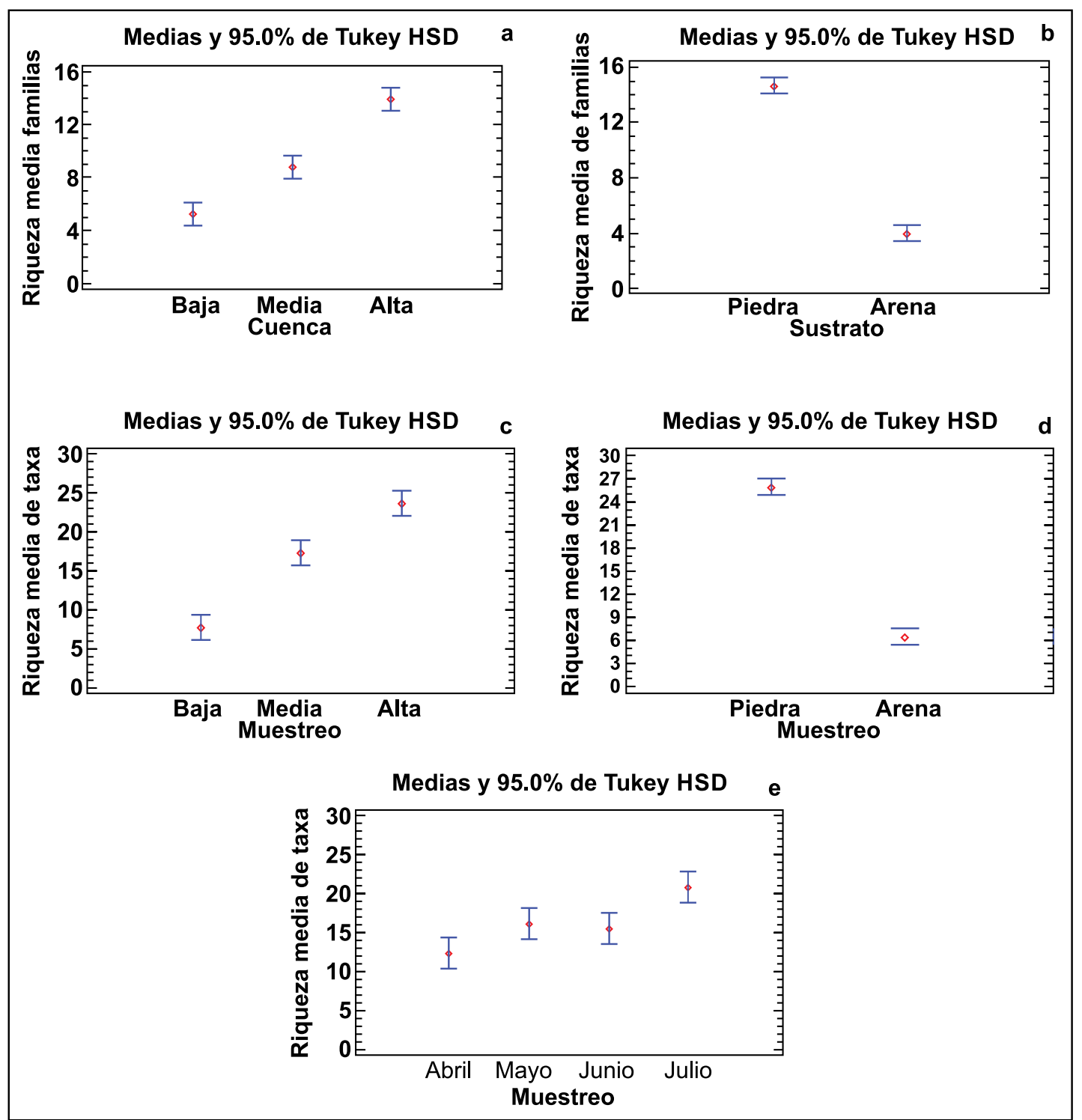

Figura $N^{\circ}$ 3: Medias e intervalos de confianza de Tukey al 95\%: a. Riqueza de familias vs. cuenca; $b$. riqueza de familias vs. sustrato; c. riqueza de taxones vs. cuenca, d. riqueza de taxones vs. sustrato; e. riqueza de taxones vs. Muestreo. 


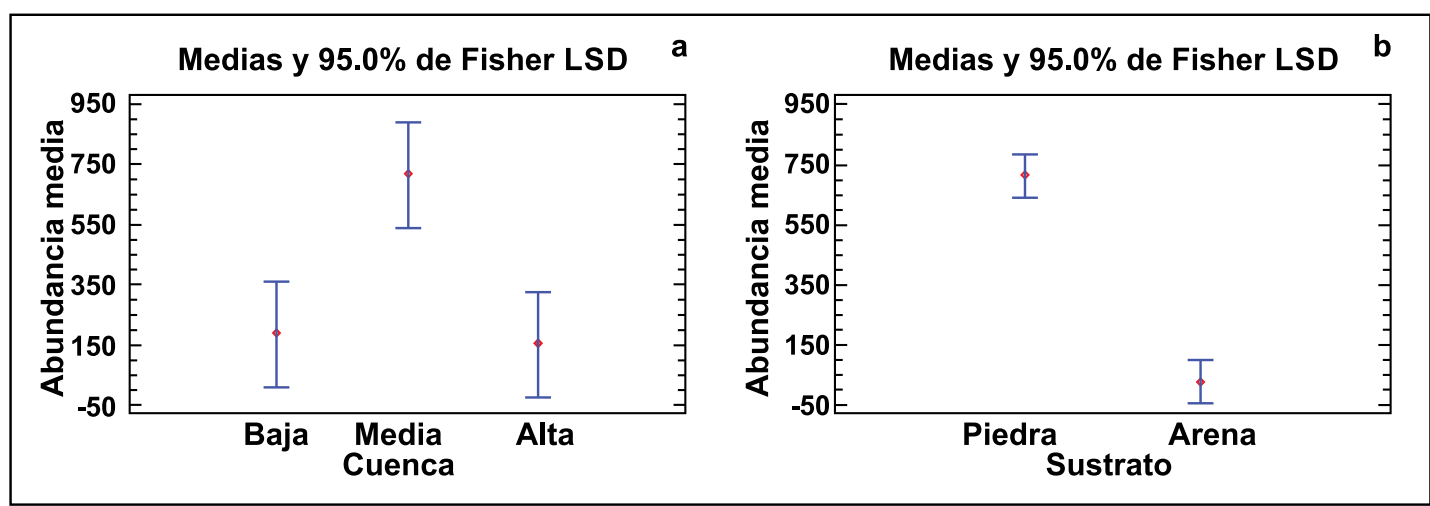

Figura $\mathrm{N}^{\circ}$ 4: Medias e intervalos de confianza de Tukey al 95\%: a. abundancia de macroinvertebrados vs. cuenca; b. abundancia de macroinvertebrados vs. sustrato.

que el de arena $(\mathrm{F}=326,98 ; \mathrm{gl}=1,71$; $\mathrm{P}=0,0001$ ) (figura $\mathrm{N}^{\circ} 3 \mathrm{~b}$ ). Por otro lado, no se observaron diferencias significativas para la misma variable entre los muestreos realizados $(F=1,22 \mathrm{gl}=3,72 ; \mathrm{P}=0,0001)$.

La riqueza media de taxones de macroinvertebrados fue mayor en la cuenca alta que en la cuenca media y la baja $(F=75,02 ; \quad g l=2,71 ; \quad P=0,0001)$ (figura $\left.N^{\circ} 3 c\right)$. Esta riqueza además fue mayor en el sustrato de piedra que en el de arena $\left(\mathrm{F}=318,64 ; \mathrm{gl}=1,71 ; \mathrm{P}=0,0001\right.$ ) (figura $\mathrm{N}^{\circ}$ $3 d)$. Por último, la misma variable mostró diferencias significativas para el caso de los muestreos ( $F=10,06 ; g l=3,72 ; P=0,0001)$ (figura $\mathrm{N}^{\circ} 3 \mathrm{e}$ ).

\section{Abundancia media de macroinvertebrados}

La abundancia media de macroinvertebrados fue mayor en la cuenca media que en la cuenca alta y la baja $(\mathrm{F}=6,68 ; \mathrm{gl}=2,71$; $\mathrm{P}=0,0026$ ) (figura $\mathrm{N}^{\circ} 4 \mathrm{a}$ ); además, la misma variable fue significativamente mayor en el sustrato de piedra que en el de arena $(F=91,31 ; g l=1,71 ; P=0,0001)$ (figura $N^{\circ} 4 b$ ).

\section{Índice Biótico Andino (IBA) y Índice de Estado Ecológico (ECOSTRIAND)}

El puntaje medio mayor del IBA se obtuvo en la cuenca alta $(177,5)$, y en la cuenca media y baja se obtuvieron puntajes de
135 y 80,3 , respectivamente. Pese a esta diferencia, las 3 cuencas se encuentran dentro del rango de "Muy buena calidad" según el IBA. Sin embargo, al combinar los índices QBR-And y el IBA, el índice ECOSTRIAND mostró una disminución de "Muy bueno" a "Regular" desde las zonas de la cuenca alta y media hacia la baja. La tabla $N^{\circ} 4$ muestra la presencia de familias bioindicadoras, según el IBA, encontradas en la microcuenca San Alberto.

\section{Composición y estructura de macroinvertebrados}

Los órdenes más abundantes de la cuenca alta fueron Trichoptera $(49,88 \%)$ y Ephemeroptera (28,58\%), con un $31,13 \%$ de la familia Leptoceridae. El 25,58\% estuvo representada por la familia Baetidae. Otras familias presentes en la cuenca alta fueron Leptohyphidae, Leptophlebiidae, Elmidae, Psephenidae, Hydroptilidae, Hydropsychidae, Glossosomatidae, Calamoceratidae, Helicopsychidae y Sericostomatidae.

En la cuenca media, en cambio, los órdenes más abundantes fueron Ephemeroptera $(53,53 \%)$ y Diptera $(41,12 \%)$, y las familias más abundantes estuvieron representadas por Baetidae (53,44\%), Chironomidae $(31,01 \%)$ y Simuliidae (9,01\%). En el caso de la cuenca baja, los órdenes con poblaciones más abundantes también fueron Ephemeroptera (53,53\%) y Diptera $(41,12 \%)$, y las familias más abundantes, estuvieron dominadas por Baetidae $(55,80 \%)$ y Chironomidae $(35,75 \%)$. 
Tabla $N^{\circ}$ 4: Lista de bioindicadores de la calidad de agua y su puntuación IBA de la microcuenca San Alberto.

\begin{tabular}{|c|c|c|c|c|}
\hline Phylum & Clase & Orden & Familia & Valor IBA \\
\hline & & Hemiptera & Veliidae & 5 \\
\hline & & Lepidoptera & Pyralidae & 4 \\
\hline & & & Leptohyphidae & 7 \\
\hline & & Ephemeroptera & Baetidae & 4 \\
\hline & & & Leptophlebiidae & 10 \\
\hline & & & Oligoneuriidae & 10 \\
\hline & & Odonata & Gomphidae & 8 \\
\hline & & & Elmidae & 5 \\
\hline & & & Psephenidae & 5 \\
\hline & & Coleoptera & Scirtidae & 5 \\
\hline & & & Ptilodactylidae & 5 \\
\hline & & & Hydrophiloidea & 3 \\
\hline & & Plecoptera & Perlidae & 10 \\
\hline & & & Hydroptilidae & 6 \\
\hline & & & Leptoceridae & 8 \\
\hline & & & Hydropsychidae & 5 \\
\hline & & & Glossosomatidae & 7 \\
\hline & Insecta & & Anomalopsychidae & 10 \\
\hline \multirow[t]{20}{*}{ Arthropoda } & & Trichoptera & Calamoceratidae & 10 \\
\hline & & & Helicopsychidae & 10 \\
\hline & & & Philopotamidae & 8 \\
\hline & & & Odontoceridae & 10 \\
\hline & & & Polycentropodidae & 8 \\
\hline & & & Xiphocentronidae & 8 \\
\hline & & & Hydrobiosidae & 8 \\
\hline & & & Blephariceridae & 10 \\
\hline & & & Ceratopogonidae & 4 \\
\hline & & & Chironomidae & 2 \\
\hline & & & Simulidae & 5 \\
\hline & & & Empididae & 4 \\
\hline & & Diptera & Psychodidae & 3 \\
\hline & & & Dixidae & 4 \\
\hline & & & Tipulidae & 5 \\
\hline & & & Stratiomyidae & 4 \\
\hline & & & Muscidae & 2 \\
\hline & & & Ephidridae & 2 \\
\hline & Arachnoidea & Acari & Acaridae & 4 \\
\hline & Crustacea & Amphipoda & Hyalellidae & 6 \\
\hline
\end{tabular}

\section{Parámetros fisicoquímicos}

Los valores medios de $\mathrm{pH}$ fueron mayores en la cuenca alta que en la media y la baja $(F=63,24 ; g l=2,71 ; P=0,0001)$. Por otro lado, los valores medios de temperatura variaron en cada zona de la cuenca y los valores fueron más bajos en la cuenca alta en comparación con la cuenca media y la baja $(F=2968,32 ; g l=2,71 ; P=0,0001)$. Los valores medios de concentración de oxígeno disuelto fueron mayores en la zona baja en comparación con la zona alta y media que no muestran mayores diferencias entre sí $(\mathrm{F}=64,76 ; \mathrm{gl}=2,71$; $\mathrm{P}=0,0001)$. Además, la concentración de oxígeno disuelto presentó un pico en mayo para luego disminuir en los meses de junio 


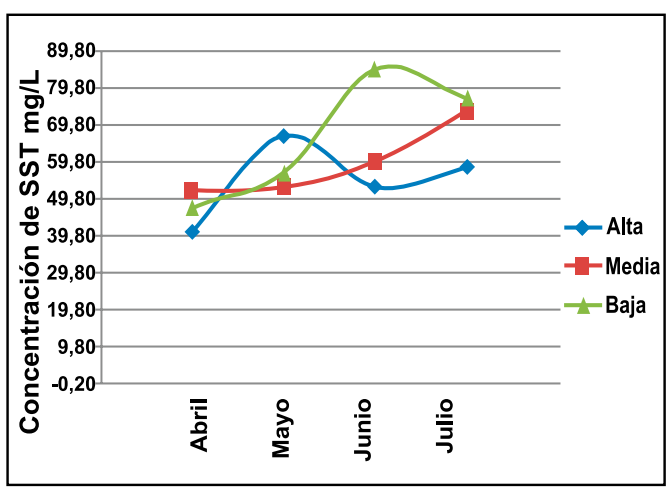

Figura $\mathrm{N}^{\circ}$ 5: Concentración de SST durante las cuatro fechas evaluadas.

$y$ julio $(F=174,61 ; g l=3,71 ; P=0,0001)$.

Los valores medios de conductividad eléctrica fueron mayores en la zona alta en comparación con la zona baja y la zona media respectivamente $(F=169,34$; $\mathrm{gl}=2,71 ; \mathrm{P}=0,0001)$. Asimismo, se observó un pico medio de conductividad eléctrica en abril en comparación con los otros muestreos realizados $(\mathrm{F}=74,51 ; \mathrm{gl}=3,71$; $P=0,0001)$. Los valores medios de sólidos totales disueltos (STD) fueron mayores en la cuenca baja que en la cuenca alta y la media $(F=488,43 ; \mathrm{gl}=2,71 ; \mathrm{P}=0,0001)$. Con respecto a cada muestreo, los valores medios de sales solubles totales (SST) mostraron notables diferencias específicamente en julio que tuvo los valores más altos en comparación con las demás muestras; los meses de mayo y junio no variaron entre ellos $(F=91,70$; $g l=3,71 ; P=0,0001$ ) (figura $N^{\circ} 5$ ). Por último, la turbidez fue mayor en la zona baja en comparación con la media y la alta que presentó los menores valores medios $(\mathrm{F}=112,58 ; \mathrm{gl}=2,71 ; \mathrm{P}=0,0001)$.

\section{Metales en el agua}

Los metales plomo, cadmio, manganeso y cromo no fueron detectados durante el período de investigación en ningún punto de muestreo. Los valores de hierro más altos fueron obtenidos en la cuenca media, llegando hasta $0,29 \mathrm{mg} / \mathrm{L}$ en junio. Valores de zinc se detectaron solo en la cuenca media en abril y en la cuenca baja en mayo. Además se registró $0,09 \mathrm{mg} / \mathrm{L}$ de cobre solo en la cuenca baja en mayo.
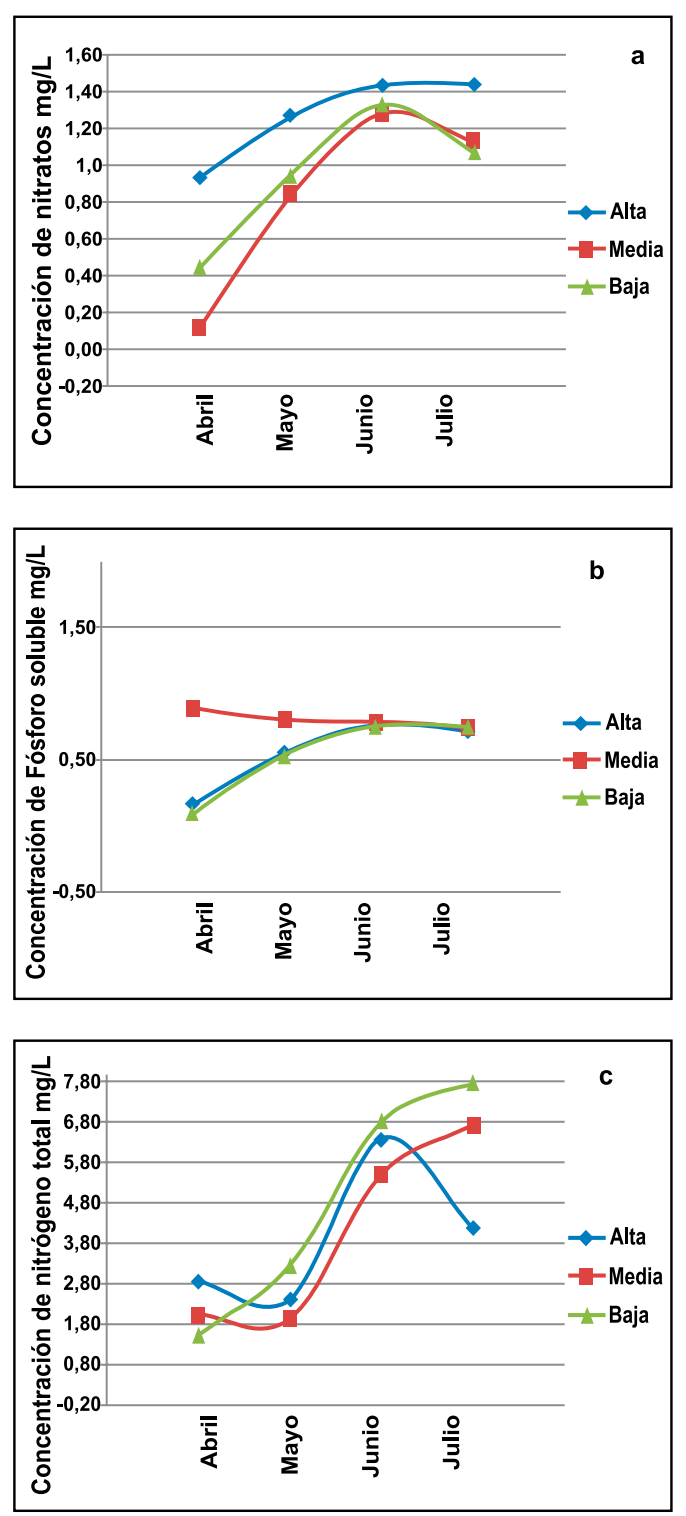

Figura $\mathrm{N}^{\circ}$ 6: Concentración de nutrientes en la cuencas alta, media y baja en las cuatro fechas evaluadas: $a$. nitratos; b. fósforo; c. nitrógeno total.

\section{Nutrientes en el agua}

La figura $\mathrm{N}^{\circ} 6$ muestra las concentraciones de los nutrientes en el agua de las 3 zonas muestreadas durante el periodo de investigación. Las concentraciones de nitratos se incrementan durante los tres primeros meses en las tres zonas de muestreo (figura $\mathrm{N}^{\circ} 5 \mathrm{a}$ ). Los valores más altos se mantienen en la cuenca alta con valores que van desde los $0,93 \mathrm{mg} / \mathrm{L}$ (abril) hasta los 1,44 mg/L (junio). Los valores de 
fósforo en la cuenca media se mantuvieron estables, mientras que en las cuencas alta y baja se mostro un ligero incremento a través del tiempo. La zona media presentó los valores más altos de fósforo soluble en el período de investigación, registrándose en abril el mayor valor, con $0,8 \mathrm{mg} / \mathrm{L}$; en tanto que los valores obtenidos en las cuencas alta y baja fueron muy similares (figura $\mathrm{N}^{\circ} 5 \mathrm{~b}$ ). Los valores de concentración de nitrógeno total a través del tiempo en las cuencas media y baja, presentaron un aumento sostenido hacia el final del estudio, excepto en el último muestreo de la zona alta cuando los valores bajaron nuevamente (figura $\mathrm{N}^{\circ} 6 \mathrm{c}$ ).

\section{DISCUSIÓN}

La heterogeneidad del hábitat fluvial es uno de los principales factores de influencia de la riqueza de especies de invertebrados acuáticos $(16,17)$. El valor alto del índice IHF para la cuenca alta indica que el hábitat fluvial alberga una comunidad de macroinvertebrados diversa, pues el hábitat proporciona fuente de alimento $y$ suministra espacio físico para las especies. La disminución de los valores del IHF y QBRAnd de las cuencas, se debe principalmente a la presencia del PNYCh en la parte alta, la misma que está protegida y preservada por las normas del Estado y a la existencia de disturbios antrópicos en la parte media y baja tales como la deforestación, la presencia de monocultivos, el arrojo de residuos sólidos, desagües y la presencia de pastizales adyacentes. Esto coincide los diseñadores de los índices, que afirman que ambos índices están estrechamente relacionados y mientras más altos son los valores QBR-And, mayores son los datos obtenidos con el IHF (4). Así pues, la vegetación riparia y la fauna bentónica se encuentran influenciadas directamente por las características físicas del cauce (geomorfología, velocidad de la corriente, tiempo de retención y tipo de sustrato) (18).
De acuerdo con los resultados, si bien los valores del IBA estimados disminuyeron al descender desde la parte alta de la cuenca a la baja, estos cambios no fueron lo suficientemente fuertes como para cambiar de rango de calidad y resultar en una menor calidad de agua. Sin embargo, de acuerdo al índice ECOSTRIAND, la calidad ecológica sí fue afectada por el cambio en las condiciones de la vegetación ribereña, al disminuir la categoría de muy bueno a regular. La presencia de las familias con puntuación 10 solo en la cuenca alta (Oligoneurridae [Ephemeroptera] representado por Lachlania sp., y Anomalopsychidae [Trichoptera] representado por Contulma sp.), indica que las aguas de esta zona de muestreo son muy limpias; además, al presentar gran diversidad de macroinvertebrados con puntuaciones altas, se sugiere su alta calidad biológica y su capacidad de albergar diversa fauna acuática. Una abundancia mucho mayor para los chironómidos y simúlidos (dípteros) en las zonas media y baja expresa que estas áreas de muestreo están expuestas a cierto grado de contaminación que puede provenir de fuentes antrópicas.

Las condiciones abióticas, las interacciones biológicas, la heterogeneidad ambiental y el microhábitat son factores que explican las comunidades presentes en un lugar determinado (19). Sitios no alterados por la actividad antrópica presentarían una abundante riqueza y diversidad taxonómica, principalmente de insectos de los órdenes Ephemeroptera, Plecóptera y Trichoptera (1). La vegetación ribereña abundante y conservada de la cabecera de la microcuenca, proporciona al río mayor cantidad de hábitats, fuentes de alimentación y refugio. Esto coincide con lo observado en la microcuenca San Alberto, debido a que en la zona alta se beneficia el desarrollo de una riqueza de taxones y familias de macroinvertebrados bentónicos mayor que en zonas con menor presencia de detritos $(20,21)$.

En cambio, en aquellos lugares donde sí existe una importante actividad antrópica, 
presentarían una menor riqueza y diversidad taxonómica y generalmente la comunidad quedaría representada por algunos dípteros, coleópteros, gusanos oligoquetos, moluscos, entre otros (1). En este caso, la mayor abundancia de macroinvertebrados en las zonas media y baja de la cuenca que estuvo representada por los ephemerópteros (Baetodes) y dípteros (chironómidos y simúlidos), podría ser causada por el incremento en la concentración de nutrientes, probablemente de los fertilizantes.

Los valores esperados del índice ACE en comparación con lo observado en los muestreos, indican que sería necesario realizar más muestreos para registrar algunos taxones raros que no se han colectado en este estudio. Sin embargo, los índices para el sustrato de piedra siempre aumentaron más que en el caso de la arena.

La estructura y composición del macrozoobentos seencuentraestrechamente relacionada con la costa del río, en términos de ingreso de energía la cual determina los cambios en el balance entre la heterotrofia y autotrofía en el continuo longitudinal del río que condiciona la dominancia de diferentes macroinvertebrados (22). La presencia de materia orgánica favorece la presencia de organismos desmenuzadores y colectores (23). A medida que aumenta su número de orden, el río se hace más ancho y profundo, el caudal aumenta y la velocidad de la corriente se hace más lenta, los sedimentos se acumulan en el fondo y la producción vegetal disminuye dando una vuelta gradual a la heterotrofía. La fuente de energía deriva del material particulado fino la cual es usada por colectores de depósito y sedimentívoros que son en este caso la comunidad dominante (23). Esto condice con que en la cuenca alta se registraron tricópteros, efemerópteros y dípteros como grupos dominantes (desmenuzadores), mientras que en las cuencas media y baja los tricópteros y los colectores disminuyeron al alejarse de la cabecera de la microcuenca.

Los macroinvertebrados bentónicos mantienen relación directa con el sustrato y la riqueza de especies, familias y abundancia de macroinvertebrados (24) y lo utilizan para desplazarse, refugiarse o alimentarse, por lo que un cambio en la composición del mismo conlleva cambios más o menos drásticos en la estructura y composición de las comunidades de macroinvertebrados (25).

De acuerdo con este estudio, el sustrato piedra proporciona mayores opciones de desarrollo a los macroinvertebrados que el sustrato arena. Losfondos arenosos albergan pocas especies, con pocos individuos por especie. Los fondos pedregosos suelen ser más ricos, en especial cuando las rocas son grandes y finalmente cuando hay vegetación la fauna es aún más diversa y difiere considerablemente de la fauna de otros sustratos (24). Del mismo modo, se afirma que las gravas y guijarros son los substratos más densamente colonizados en todos los ecosistemas acuáticos (26). Esto coincide con lo observado en el presente estudio, en el cual las diferencias entre las variables biológicas (biodiversidad, composición y estructura) asociadas al sustrato fueron evidentes en la investigación donde la cuenca alta estuvo compuesta en mayor grado por bloques y piedras (con mayor riqueza de taxones), mientras que la zona baja, por cantos, grava y arena (con menor riqueza de taxones).

Las diversas actividades antrópicas pueden afectar a los cursos de agua generando cambios drásticos en las comunidades de macroinvertebrados (27). La diferencia de pH podría deberse a la contaminación por residuos orgánicos e inorgánicos domésticos y productos agrícolas introducidos al medio acuático en las cuencas media y baja. La diferencia de temperatura está ligada directamente a la altitud de la zona. La gradiente altitudinal del PNYCh y la abundante vegetación ribereña de la cuenca alta proporciona mayor sombra al río, impidiendo que la temperatura aumente. Por otro lado, la variación del \% de oxígeno disuelto se debería a la mayor concentración de nitratos y sales solubles totales que agotan el oxígeno del agua en 
sus procesos químicos. Sin embargo, en los tres puntos de muestreo la concentración de oxígeno se mantuvo por los valores óptimos para este tipo de ambientes.

La diferencia de conductividad eléctrica de las cuencas evaluadas se basa en sus respectivas concentraciones de sólidos totales disueltos y de sales solubles totales, por lo que un aumento de conductividad se debe a un aumento delas variables $(6,28)$. Un aumento de la conductividad eléctrica en la zona baja, disminuye significativamente la riqueza de taxones y familia de macroinvertebrados bentónicos, excepto para algunos grupos que se benefician con los sólidos en suspensión tales como los chironómidos y simúlidos (29). Los simúlidos se han especializado en la explotación de partículas en suspensión en el agua, para lo cual utilizan estructuras cefálicas y secreciones (30) y remueven partículas transportadas a lo largo del río, concentrándolas en forma de heces y dejándolas disponibles a otros grupos de organismos. En el presente estudio, la gran abundancia de chironómidos en las cuencas media y baja ha sido favorecida por el aumento de partículas en suspensión, coincidiendo con un aumento de áreas ribereñas desprotegidas y sin vegetación original.

Por su parte, la diferencia entre los valores de turbidez en las zonas de muestreo de las tres zonas de la cuenca, fue probablemente causada por la actividad con maquinaria pesada aguas arriba de la parte baja, que provoca la remoción y arrastre de sedimentos. Además, la poca cobertura boscosa que caracteriza la zona baja por actividades agrícolas, de deforestación y ganadería, hace que el suelo se erosione con las lluvias y al no haber estabilidad adecuada en las orillas del río, permite que los sedimentos lleguen al mismo (3).

Los nitratos, fósforo soluble y nitrógeno total aumentan en el tiempo, posiblemente debido a la menor dilución de los nutrientes en la época seca (31) aunque los agricultores pueden haber usado más fertilizantes al final del presente estudio. La mayor cantidad de nitratos en la cuenca alta puede deberse a la mayor materia orgánica descompuesta (hojarasca, troncos, ramas y raíces) de los bosques de ribera conservados. En épocas seca y en ambientes no contaminados, se deberían registrar concentraciones de fósforo entre 0.05 y $0.1 \mathrm{mg} / \mathrm{L}$ (30). En la zona media hay una fuente adicional de fósforo posiblemente humana por el uso de fertilizantes agrícolas (para cultivos de granadilla), perturbación del suelo y desechos animales.

Las principales conclusiones establecidas son:

- La calidad del hábitat fluvial y de la vegetación de ribera estuvo estrechamente relacionada con la actividad antrópica dentro y fuera del PNYCh.

- La vegetación ribereña de la cuenca alta, dentro del PNYCh, es considerada de "calidad muy buena"; la vegetación de ribera de la cuenca media, de "calidad buena", y de la cuenca baja, de "calidad mala", con diversos grados de perturbación.

- El índice biótico andino mostró que la calidad del agua en la cuenca alta, media y baja fue "muy buena", pero al combinarse con el QBR-And y aplicar el índice ECOSTRIAND, la cuenca alta y media obtuvieron un estado ecológico del río "muy bueno" mientras que la cuenca baja cambió a un estado ecológico "regular".

- Los grupos más intolerantes a la contaminación se registraron principalmente y en mayor abundancia en la cuenca alta (Leptophlebiidae, Oligoneuriidae, Perlidae, Anomalopsychidae, Calamoceratidae, Helicopsychidae, Odontoceridae, Blephariceridae). Los grupos tolerantes de Chironomidae y Baetidae dominaron las cuencas media y baja, que se caracterizan por ser lugares perturbados.

- La cuenca alta presenta mayor heterogeneidad y fuente de alimentación 
y refugio para el adecuado desarrollo de macroinvertebrados bentónicos.

- La riqueza total de taxones de macroinvertebrados bentónicos estuvo condicionada por la zona de la microcuenca de San Alberto. Tal riqueza fue mayor en la parte alta que en la media y la baja. La vegetación ribereña más conservada y de mejor calidad de hábitat fluvial también ocurrió en la cuenca alta, mientras que las cuencas media y baja fueron afectadas por perturbaciones humanas.

- La abundancia total de macroinvertebrados fue mayor en la cuenca media, probablemente por una mayor concentración de nutrientes provenientes de la aplicación de fertilizantes.

- El método de colecta por sustrato de piedra fue el que permitió colectar mayor cantidad de taxones que el método por sustrato de arena.

- Este estudio permitió aplicar cuatro índices y plantea un inicio para el uso de los mismos en zonas cercanas para evaluar la calidad de agua y hábitat en la zona.

\section{REFERENCIAS BIBLIOGRÁFICAS}

1. Jara C. Evaluación de la existencia de insectos bioindicadores de la calidad del agua en zonas ritrónicas y potámicas de tres ríos de la zona semiárida de Chile [tesis]. Chile: Universidad de Chile, Facultad de Ciencias; 2002.

2. Instituto Nacional de Recursos Naturales. Plan Maestro 2005 - 2009, Parque Nacional Yanachaga Chemillén. Lima: INRENA, Intendencia de Áreas Naturales Protegidas; 2005.

3. Roldán G. Bioindicación de la calidad del agua en Colombia. Uso del método BMWP/Col. 1a ed. Colombia: Universidad de Antioquia; 2003.

4. Acosta R, Ríos B, Rieradevall M, Prat N. Propuesta de un protocolo de evaluación de la calidad ecológica de ríos andinos (CERA) y su aplicación a dos cuencas en Ecuador y Perú. Limnética. 2009; 28 1: 35-64.

5. Metcalfe JL. Biological waterquality assessment of rivers: use of macroinvertebrate communities. En: C. Peter \& E.P. Jeoffrey, editors. The River Handbook: hydrological and ecological principles. Oxford: Blackwell Scientific Publications Press; 1994. p. 145-170.

6. Roldán G. Los Macroinvertebrados y su valor como indicadores de la calidad del agua. Revista Acad. Colomb. Ci. Exact. 1999; 23(88): 375-387.

7. Neumann $M$, Liess $M$, Ralf $S$. An expert system to estimate the pesticide contamination of small streams using benthic macroinvertebrates as bioindicators. Knowledge base of limpact. Ecological indicators; 2003.

8. United States Environmental Protection Agency. Lake and Reservoir Bioassessment and Biocriteria: Technical Guidance Document. Washington DC: U.S. Environmental Protection Agency; 2003.

9. Catchpole D. The ecology of vascular epiphytes on a Ficus L. host (Moraceae) in a Peruvian cloud forest [dissertation]. Australia: University of Tasmania, School of Geography and Environmental Studies; 2004.

10. Holdridge LR. Life zone ecology. San José: CR. Tropical. Science. Center; 1967.

11. Quispe A, Tello J. Especies forestales de uso múltiple de los bosques de neblina en el nororiente del Perú. $1^{\mathfrak{a}}$ ed. Lima: Fondebosque; 2001.

12. Pardo I, Àlvarez M, Moreno JL, Vivas S, Bonada $N$, et al. El hábitat de los ríos Mediterráneos. Diseño de un índice de diversidad de hábitat. Limnetica. 2002; 21 (3-4): 115-134.

13. Roldán G. Guía para el estudio de los macroinvertebrados acuáticos del departamento de Antioquia. $1^{\mathrm{a}}$ ed. Colombia: Fondo FEN Conciencias, 
Universidad de Antioquia; 1988.

14. Merritt RW, Cummins KW, Berg MB. An Introduction to the aquatic insect of North America. Fourth edition. USA: Kendall/Hunt publishing company; 2008.

15. Domínguez E， Fernández HR. Macroinvertebrados bentónicos sudamericanos. Sistemática y biología. $1^{\mathrm{a}}$ ed. Argentina: Fundación Miguel Lillo; 2009.

16. Voelz NJ, McArthur JV. An exploration of factors influencing lotic insect species richness. Biodiversity and Conservation. 2000; 9:1543-1570.

17. Gregory SV, Swanson FJ, McKee WA, Cummins KW. An ecosystem perspective of riparian zones. BioScience. 1991; 41, 540-551.

18. Márquez EJ, Fariñas MR, Briceño $B$, Rada FJ. Dsitribution of grasses along an altitudinal gradient in a Venezuelan paramo. Rev. Chil. Hist. Nat. 2001; 77 : 649-660.

19. Poof NL. Landscape filters and species traits: toward mechanistic understanding and prediction in stream ecology. J N AM BENTHOL SOC. 1997; 16(2): 391409.

20. Siccama TG, Borman FH, Likens GE. The Hubbard Brook Ecosystem Study: Productivity. Nutrients, and Phytosociology of the Herbaceous layer. Ecological Monographs. 1970; 40: 389-402.

21. Covich AP. Geographical and historical comparisons of neotropical streams biotic diversity and detrital processing in highly variable habitats. J N AM BENTHOL SOC. 1988; 7:361-386.

22. Cummnig K, Klug J. Feeding ecology of stream invertebrates. Annu. Rev. Ecol. Syst,. 1979; 10: 147-172.

23. Vannote R, Minshall G, Cummins K, Sedell R, Cushing C. The River Continium concept. Can J. Fish. Aquat. Sci. 1980; 37: 130-137.

24. Rivera R. Estructura y composición de la comunidad de macroinvertebrados bentónicos en ríos de páramo y zonas boscosas, en los andes venezolanos [tesis]. Merida: Universidad de los Andes; 2004.
25. Álvarez M. Estudio de la variabilidad espacio-temporal de las comunidades de macroinvertebrados bentónicos en los ecosistemas fluviales de Cantabria. Repercusiones para la aplicación de la directiva macro del agua [tesis doctoral]. Santander: Universidad de Cantabria, Departamento de Ciencias y Técnicas del Agua y del Medio Ambiente; 2009.

26. Hynes HB. The ecology of running waters. $1^{\mathrm{a}}$ ed. Toronto: University of Toronto Press; 1970.

27. Ortiz JD, Marti E, Puig MA. Recovery of the macroinvertebrate community below a wastewater treatment plant input in a Mediterranean stream. Hydrobiologia. 2005; 545: 289-302.

28. Calderón J. Evaluación de la comunidad de macroinvertebrados bentónicos y la calidad fisicoquímica del agua en la parte alta de la quebrada el Carracá del municipio de los Santos [tesis doctoral]. Santander: Universidad Industrial de Santander; 2004.

29. Callisto $M$, Moreno $M$, Barbosa $F$. Hábitat Diversity and Benthic Functional Trophic Groups at Serra Do Cipó Southeast Brazil. Revista Brasil. Biol. 1999; 61(2): 259- 266.

30. Margalef R. Limnología. $1^{\text {a }}$ ed. Barcelona: Omega; 1983.

31. Gibbs RJ. Mechanisms controlling world water chemistry. Science. 1970; 170:1088-1090. 